\section{High Voltage Scanning Electron Microscopy Theory and Applications}

Mark Rigler \& William Longo

Materials Analytical Services, Inc.

A variety of energy emissions occur as a result of primary beam interaction with the specimen surface. Secondary electrons, X-rays, visible photons, near IR photons, and Auger electrons are emitted during inelastic scattering of electrons. Backscattered electrons (BSE) are emitted during elastic scattering of primary electrons. Backscattered electrons are those electrons which pass through the electron cloud of an atom and change direction without much energy loss. BSEs may diffuse into the sample or may escape from the sample surface. In practice, the primary electron beam penetrates deeply into low $Z$ (atomic number) materials and produces few BSEs while high $Z$ materials retard primary beam penetration and emit large numbers of BSEs. According to Murata et al.,' the higher the atomic number, the smaller the mean free path between electron scattering events (i.e. $528 \mathrm{~A}$ for $\mathrm{Al}$ vs. $50 \mathrm{~A}$ for $\mathrm{Au}$ at $30 \mathrm{KeV}$ ) and the higher the probability of scattering. In practicality, the contrast of the material in the backscatter mode is dependant upon $Z$. The simplicity of this relationship is the basis for the operation of SEMs using a BSE detector.

For samples with low Z materials overlaying higher Z materials (such as semiconductors), why not use electron depth penetration and the resulting BSE electrons as a way to accurately visualize subsurface features without sample preparation? If the primary electron beam could be scanned over the surface at voltages in excess of $30 \mathrm{KeV}$, BSE emissions should be available from successively deeper regions of the sample as the primary beam voltage is increased. This concept has been proven in STEMs at voltages exceeding $100 \mathrm{KeV}^{2}$ however, the STEM imposes severe restrictions on sample size ( $\leq 3.0 \mathrm{~mm}$ diameter) and restricts the type of test that can be performed due to the limited amount of working area in a typical STEM sample holder

High voltage SEMS (HVSEM) with large specimen chambers are the answer to sample size problems and can provide working room for real time tests of wafer arrays of microcircuits. In a March, 1994 Semiconductor international article, high energy electron beam tools (100-200 KeV) were rated number five on a list of significant trends in SEM ${ }^{3}$ due to their ability to vișualize components on intact semiconductors. The basic HVSEM system consists of a scanning high voltage beam $(40-200 \mathrm{KeV})$, large specimen chamber containing a special BSE detector ${ }_{1}$ and a precision stage to accommodate a semiconductor wafer or probe card assembly. The BSE detector is mounted $90^{\circ}$ to the specimen surface at a working distance of $2-3 \mathrm{~mm}$ Presently, Hitachi and Materials Analytical Services, Inc. ${ }^{4}$ build HVSEMs.

HVSEM resolution depends upon accelerating voltage and material type. Figure 1 shows BSE images from successive views deeper into a glass covered modern computer chip. At $40 \mathrm{KeV}$, the depth of penetration through the glass passivation layer on the surface is approximately 1-3 $\mu \mathrm{m}$ and the first layer of metallization is visible. At $100 \mathrm{KeV}$, the second level of

\section{MATERIALS GROUP LEADER POSITION AVAILABLE}

Materials Analytical Services, Inc., a leading materials analysis lab, has an immediate opening at our Atlanta facility for a Materials Group Leader. The selected candidate will have either a MS or Ph.D. in a related field with 3-4 years experience in failure analysis and general materials characterization using both optical and electron microscopy techniques.

All inquiries will be kept confidential. Submit your resume to Materials Analytical Services, 3597 Parkway Lane, Suite 250, Norcross, GA 30092, Atten.: Human Resources.

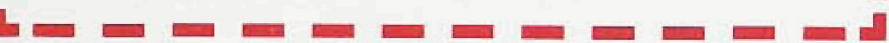

metallization, approximately $6-8 \mu \mathrm{m}$ below the surface, is visible (even through the top metal layer). Additionally, tungsten vias which bridge the metal line layers are visible (white dots) as the voltage is increased.

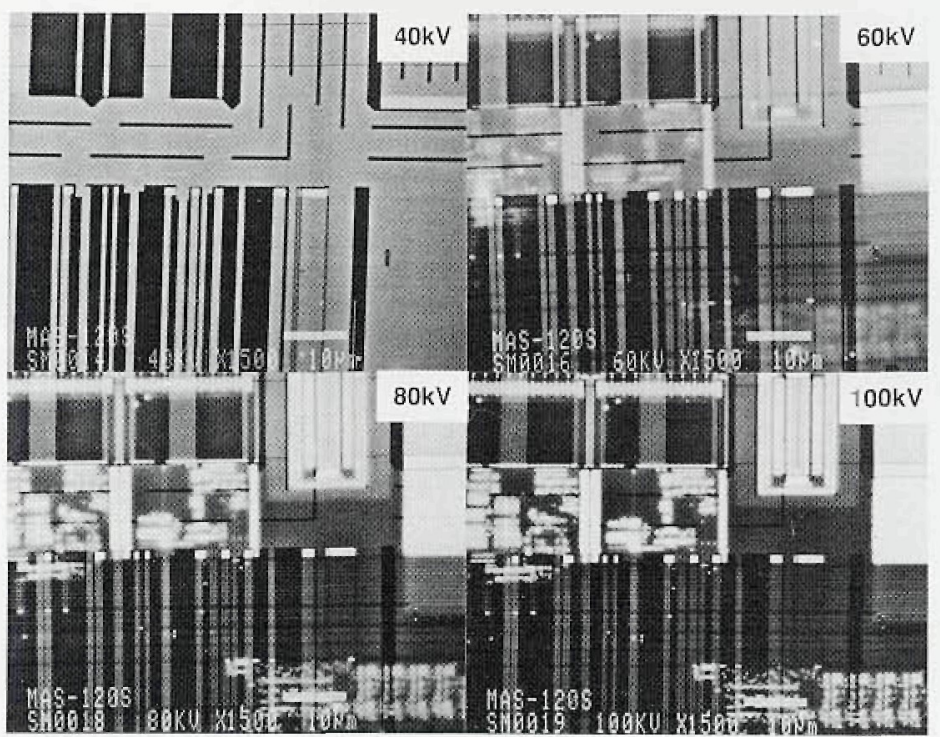

Figure 1: Successively deeper views into a computer chip. 120s HVSEM

The need for HVSEMs has been driven by semiconductor manufacturers and researchers who want to understand problems associated with electromigration. According to Thompson and Lloyd ${ }_{4}^{5}$ current densities measured in amps per $\mathrm{cm}^{2}$ in integrated circuit (IC) devices with micron size metal lines can be 400 times higher than current densities in house wiring. Electromigration occurs when the momentum of electrons moving through micro miniature metal lines causes the atoms in those lines to "move". Metal movement causes holes (voids) in the lines and stress at contact points (Figure 2). Ultimately, circuit line failure can occur when the voids become too large. Presently, the only way to study electromigration is by cross sectioning and polishing the circuit or by etching away the glass. In this type of preparation, the specimen is usually destroyed and the actual cause of the defect may be caused by the prep itself. Using the HVSEM however, the lines below the surface glass can be visualized in real time, even while the circuit is thermally or electrically stressed. Additional uses for the HVSEM include: visualizing fuses, gates, arrays on intact IC wafers or chips, locating film inclusions, defects, and submicron contaminants. Additionally, the HVSEM could be used to examine inclusions and defects in ceramic materials, plastics, and biological specimens that are below the surface.

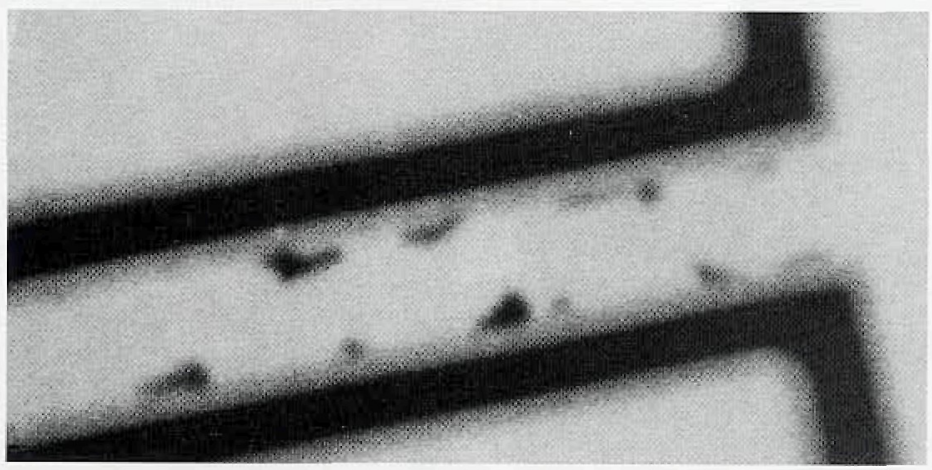

Figure 2. Line voids (irregular dark patches on bright metal lines) 120 s HVSEM, $100 \mathrm{KeV} \mathrm{Bar}=1 \mu \mathrm{m}$

1. K. Murate, T. Matsukawa \& R. Shimizu, Jap. J. App Physics, 10, 678, (1971)

2. P.A. Flinn, A.S. Mack, P.R. Besser \& T.N. Marieb, Mat. Res. Soc. Bulletin 12,19 (1994).

3. L. Peters, Semicon. Int. $\underline{3}, 50$ (1994)

4. PE. Russell, A. McCanna, W. E. Longo \& M. Madden Proc Microsc. Soc Amer. in Press (1994)

5. Thompson, CV \& Lloyd, J.R., Mat. Res. Soc. Bull 12, 18, 19 (1993) 


\section{Severe Environment, High Vacuum, Soft X-Ray Window}

Does your SEM/TEM/Spectrometer eat light element windows for lunch?

Too Hot? $\cdot$ Too Wet? $\cdot$ Too Dirty? $\cdot$ Too Corrosive?

\section{Ask your Spectrometer Vendor to} use the DB1.2 Window from Moxtek!

- Up to $30 \mathrm{~mm}^{2}$ active area

- Bakeable to $200^{\circ} \mathrm{C}$

- Chemically Resistant

- Super Strong

- 50\% transmission at Beryllium $K \alpha$

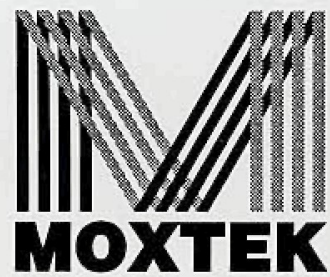

452 West 1260 North, Orem, UT 84057 U.S.A. Phone (801) 225-0930 - Fax (801) 221-1121.

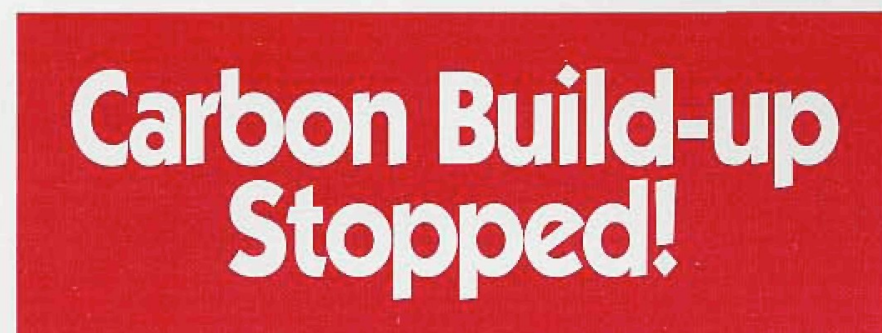

\section{Applications Lab Secret Revealed:}
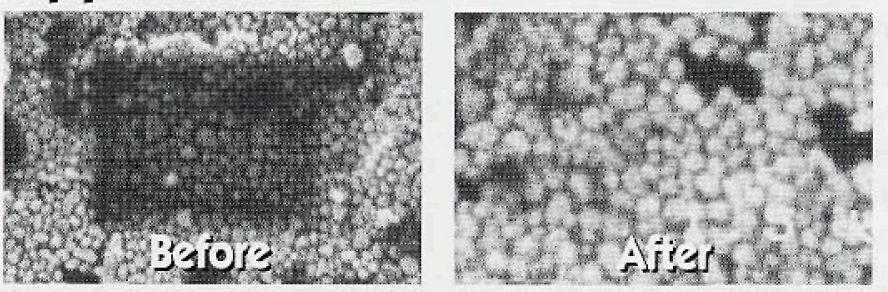

SEM manufacturers won't admit it, but most SEMs are subject to contamination build-up-even dry pumped systems. To stop hydrocarbon condensation, major applications labs and SEM users rely on the XEI Scientific SEM-CLEAN ${ }^{\text {mo }}$ system.

Result: Outstanding pictures at low $\mathrm{kV}$ and high resolution and no oi on EDS X-ray detector windows. The Nitrogen purge of the inexpensive SEM-CLEAN system cleans your electron microscope while you're away.

\section{SEM-CLEAN'" Stops the Oil}

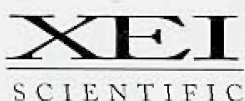

3124 Wessex Way, Redwood City, CA 94061 - 415-369-0133 - Fax 415-363-1659

\section{Microdroplets of Liquid}

Part l: Glass Fiber Brush

Walter C. McCrone, McCrone Research Institute*

It is very important to be able to deliver tiny droplets of liquid onto any surface. There are two general methods for this. One, a glass fiber brush sealed into a capillary; and, the other, a polyethylene micropipette. Both are capable of delivering droplets as small as $100 \mu \mathrm{m}$ in diameter. Description of the glass fiber brush follows. Part II, later in this publication, will describe the polyethylene micropipette.

We have borrowed this clever idea from Fred Schneider of IBM (private communication). A bundle of 20 to 30 glass fibers, 5 to $8 \mathrm{~mm}$ long and about $20 \mu \mathrm{m}$ in diameter, is sealed with a microflame into one end of a glass capillary. A melting point capillary 1 to $2 \mathrm{~mm}$ in diameter and open at both ends is convenient (Fig. 1). To use for small drop deposition, the capillary is partly filled (2 to $3 \mathrm{~cm}$. long column of the desired liquid) so that the sealed-in end of the glass fiber bundle is fully immersed. Droplets can then be "painted" onto any surface by touching the glass fiber "brush" to the desired spot. Drop size depends on the number and size of glass fibers, the liquid head in the capillary, the splaying of the fiber brush on the surface and any movement of the brush across the surface.

Normally, the glass fiber brush is used with pure liquids because evaporation concentrates a solution on the brush and may clog the fibers. This effect can be alleviated if the solution-filled capillary is stored with its brush tip

\section{Approximate true size}

\section{Enlarged tip}

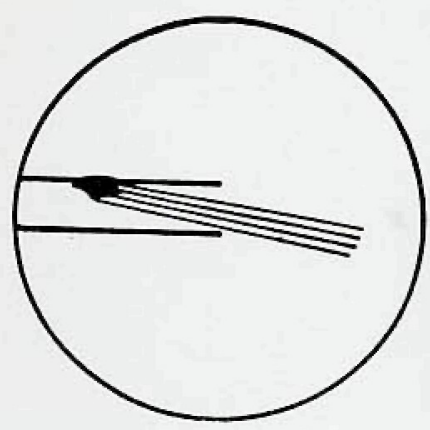

Figure 1: Glass fiber brush for deposition of microdrops

in a bottle or test tube containing a little of the appropriate solvent soaked into a cotton ball at the bottom (Fig. 2). Evaporation from the open end of the capillary is very slow, negligible over a period of several hours, and can be minimized further by drawing down the open end of the capillary to a very fine opening. In this way, a dilute solution of, say, collodion can be kept ready for

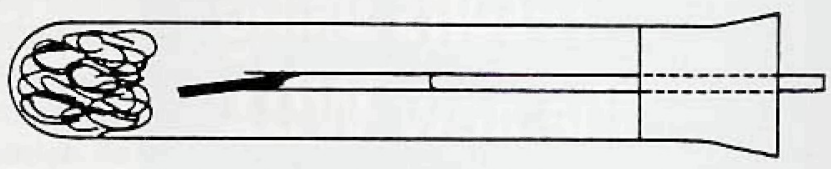

Figure 2: Protective container for glass fiber brush; a glass wool plug in the bottom of the test tube is soaked with solvent

instant use for days and even weeks. It is essential that the brush end of the capillary be stored in the saturated solvent vapor corresponding to the solution used. Strictly speaking, one would avoid small concentration changes due to differences in vapor pressure if the brush tip is stored over the same solution rather than over the pure solvent.

Reprinted from The Particle Atlas, Volume 1, available from McCrone Research Institute, 2820 S. Michigan Ave., Chicago, IL 60616, Tel.: (312)8427100. The entire six-volume Particle Atlas is also available on CD-ROM. 